\title{
Supply Chain Risk Management (SCRM): uma revisão sistemática da literatura
}

A Gestão de Riscos na Cadeia de Suprimentos (SCRM) é considerada uma forma de atingir vantagem competitiva, uma vez que se baseia em sua habilidade de encarar e de se adaptar aos ambientes complexos e que expõe às Cadeias de Suprimentos a riscos constantes. No Brasil, SCRM é um tema relativamente novo, onde quase não publicações na base de dados da Scielo. Assim, esse artigo possui como objetivo principal tornar o assunto mais explícito e conhecido no Brasil, de forma que esse tema seja de conhecimento de profissionais, pesquisadores e acadêmicos. Metodologicamente a pesquisa é suportada por uma Revisão Sistemática da Literatura. O presente material traz uma revisão bibliográfica, considerando artigos de 2004 (ano da primeira publicação sobre o tema no mundo) a 2015. Partindo de uma revisão sobre Risco, Gerenciamento de Risco, Cadeia de Suprimentos, Gerenciamento da Cadeia até chegar em SCRM, apresenta-se o seu conceito, histórico, relevância e faz-se uma compilação sobre os modelos de gerenciamento de riscos apresentados. Como parte dos resultados, observaram-se divergências dos autores pesquisados sobre a classificação dos riscos na cadeia e os modelos para gerenciá-los. A relevância do tema está na capacidade de minimização de prejuízos de grandes cifras na Cadeia de Suprimentos das organizações com base na administração dos conceitos aqui abordados.

Palavras-chave: Gerenciamento de Risco; Cadeia de Suprimentos; SCRM.

\section{Supply Chain Risk Management (SCRM): a systematic literature review}

\begin{abstract}
Supply Chain Risk Management (SCRM) is considered a way to achieve competitive advantage because it relies on its ability to face and adapt to complex environments and exposes Supply Chains to constant risks. In Brazil, SCRM is a relatively new topic, where there are almost no publications in the Scielo database. Thus, this article has as main objective to make the subject more explicit and known in Brazil, so that this topic is known to professionals, researchers and academics. Methodologically the research is supported by a Systematic Review of Literature. The present material presents a bibliographic review, considering articles from 2004 (year of the first publication on the subject in the world) to 2015. Starting from a review on Risk, Risk Management, Supply Chain, Chain Management until arriving at SCRM, presents its concept, history, relevance and a compilation of the presented risk management models. As part of the results, we observed divergences from the researched authors about the classification of risks in the chain and the models to manage them. The relevance of the topic lies in the ability to minimize the losses of large numbers in the Supply Chain of organizations based on the management of the concepts discussed here.
\end{abstract}

Keywords: Risk management; Supply chain; SCRM

\section{Topic: Sistemas Logísticos}

Reviewed anonymously in the process of blind peer.

Gabriela Costa Dias

Universidade Federal Fluminense, Brasil

http://lattes.cnpq.br/0614830979000841

gabsdias@hotmail.com

Aldara Da Silva Cesar

Universidade Federal Fluminense, Brasil

http://lattes.cnpq.br/7269278746075136

aldaracesar@id.uff.br

Ualison Rébula Oliveira

Universidade Federal Fluminense, Brasil

http://lattes.cnpq.br/6460931837390456

ualison.oliveira@gmail.com
Received: 17/05/2017

Approved: 17/07/2017
Referencing this:

DIAS, G. C.; CESAR, A. S.; OLIVEIRA, U. R.. Supply Chain Risk Management (SCRM): uma revisão sistemática da literatura. Revista Brasileira de Administração Científica, v.8, n.2, p.190-207, 2017. DOI: http://doi.org/10.6008/SPC2179-684X.2017.002.0014 


\section{INTRODUÇÃO}

O ambiente externo influencia as opções estratégicas de uma empresa e suas decisões. A soma de seu entendimento sobre o ambiente externo somado ao conhecimento do ambiente interno formam sua missão, visão e auxiliam na tomada de decisão que resultem em vantagem competitiva e retornos acima da média (HITT et al., 2008). Entretanto, conforme Silva et al. (2007), o ambiente competitivo atual passou por uma reestruturação, de forma que a competição passou a ocorrer entre cadeias e não entre empresas isoladas. Para alcançar vantagem competitiva faz-se necessário o alinhamento e gerenciamento da cadeia, seu fluxo bidirecional de materiais e informação, além de relações cooperativas, pautadas em parcerias.

Dada a complexidade da própria estrutura da cadeia e do ambiente externo, esse gerenciamento deve compreender também os riscos desse ambiente à cadeia: a vantagem competitiva está baseada em sua habilidade de encarar e se adaptar aos ambientes de mudança aos quais estão expostas (TRKMAN et al., 2009). Hendricks et al. (2009) cita pesquisa com 151 executivos, dos quais $73 \%$ indicaram que suas companhias experimentaram rupturas em seus fluxos de fornecimento e $48 \%$ esperam que o risco aumente nos próximos três anos.

A vulnerabilidade e exposição ao risco das CS foram aumentadas devido a fatores como a globalização, a interdependência cada vez maior entre as empresas, o tamanho e complexidade das cadeias, ciclo de vida dos produtos cada vez menores e estratégias como Lean Six Sigma e Just in Time, além de desastres naturais como o Furacão Katrina, nos Estados Unidos, em 2005 e o tsunami na Tailândia em 2004, bem como desastres causados pelo homem, como os atentados terroristas em 2001, nos Estados Unidos.

O Gerenciamento de Risco da Cadeia de Suprimentos é um assunto relativamente novo, cujas primeiras publicações datam 2004. Contudo, a discussão dessa temática vem crescendo em âmbito internacional, que pode ser percebido pelo número de publicações ao longo da última década (Web of Science).

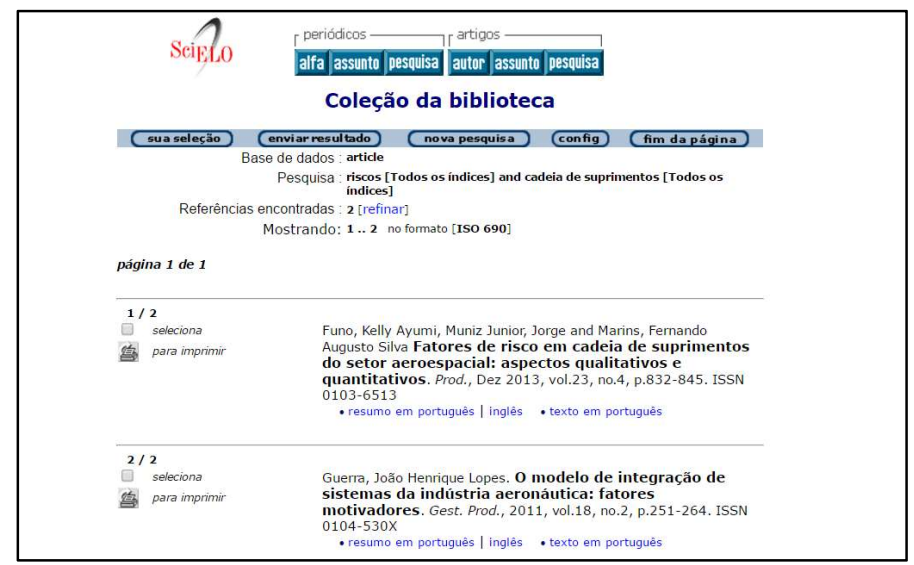

Figura 1: Resultado de buscas na base Scielo.

Entretanto, ao realizar uma busca na base Scielo, em novembro de 2016, por 'Gerenciamento de Risco' ou 'Gestão de Risco' e 'Cadeia de Suprimentos', em todos os índices, encontraram-se apenas dois artigos, porém um único relacionado ao tema da presente pesquisa, dos autores Funo et al. (2013). Isso ilustra a falta de publicações sobre SCRM no Brasil. Ressalta-se, também, que existem outros trabalhos sobre 
Gestão de Riscos em Manufatura, tal como o de Oliveira et al. (2014), porém esses não são focados em Cadeias de Suprimentos, conforme se nota na figura 1.

Quanto aos objetivos, o trabalho busca explicitar o tema no Brasil, auxiliando os pesquisadores do tema com a revisão bibliográfica, que conta com uma compilação de modelos de gerenciamento de risco da cadeia apresentados na literatura, além de um quadro com a evolução das publicações no decorrer dos anos. Ainda, auxiliando os profissionais, também explicitando o assunto que pode servir como ferramenta para estudos e reestruturações nas organizações. Sobre a justificativa e suas contribuições, o trabalho apresenta dois pontos não encontrados nas demais revisões bibliográficas de SCRM: um quadro com a evolução histórica do tema, com o ano de publicação, os autores e um breve resumo do que é tratado no mesmo, com o objetivo de situar os pesquisadores em suas pesquisas; e uma comparação dos modelos de gerenciamento de risco utilizados por cada autor, considerado um ponto de discordância dentro do tema.

O restante do artigo está organizado da seguinte maneira: a seção seguinte traz a revisão bibliográfica, escopo e objetivo principal, que contempla os assuntos 'Risco', 'Gerenciamento de Risco' apresentando seu conceito e modelo, 'Cadeia de Suprimentos', também considerando os riscos na Cadeia e classificação desses riscos, 'Gerenciamento da Cadeia de Suprimentos' até chegar no Gerenciamento de Risco da Cadeia de Suprimentos, que traz seu conceito, histórico, relevância, principais motivos que levaram ao estudo do tema, seguida de uma compilação dos modelos de SCRM na literatura e um quadro cujo objetivo é mostrar a evolução do tema, a partir das publicações a cada ano. Por fim, faz-se uma análise dos resultados e uma conclusão.

\section{REVISÃO TEÓRICA}

Risco

Essa seção é a principal do artigo, uma vez que se seu objetivo é apresentar uma revisão bibliográfica sobre o tema. O risco tem uma infinidade de conceituações dependendo do campo em que se pesquisa e que são encontradas literaturas sobre risco nos campos de finanças, marketing, gestão. e psicologia (WAGNER et al., 2008). Outra característica apresentada por Pfohl et al. (2010) é que há uma constante dicotomia entre o risco ser puramente negativo ou uma oportunidade. Especificamente para os fins de risco na CS, o risco é majoritariamente percebido, na literatura, por suas indesejáveis consequências, ou seja, por seu caráter negativo e, por isso, a importância de seu gerenciamento.

Considera-se que o risco tem dois componentes: potenciais perdas e probabilidade dessas perdas (TAKATA et al., 2013). Dessa forma, o risco pode ser entendido como o resultado esperado de um evento incerto. Essa associação com a probabilidade pode ser explicada pela derivação do estudo de risco. De acordo com Khan et al. (2007), o estudo de risco inicia na área da matemática e levou ao desenvolvimento da teoria da probabilidade. Trkman et al. (2009) e Ritchie et al. (2007a) corroboram com esses dois componentes do risco e acrescentam um terceiro: o caminho que leva a esse evento incerto. Por conseguinte, Ritchie et al. (2007b) acrescenta ainda um quarto componente, sendo esse, a percepção de exposição ao risco. Norrman e Jansson (2004) utilizam uma definição condizente com os autores anteriores de que o risco seria o nível de 
exposição às incertezas que uma companhia deve compreender e efetivamente gerenciar. Rao et al. (2009) acrescentam que a mera presença de um desses componentes não é suficiente para configurar o risco.

Já Zsidisin (2004), traz uma definição parcialmente diferente, afirmando que o risco existe quando há uma probabilidade relativamente alta de um evento prejudicial acontecer e esse evento está associado a um significante impacto ou custo. E Hallikas (2004) utiliza uma abordagem da Teoria da Utilidade para definir risco como perda da utilidade esperada. Assemelhando-se a essa relação proposta, Rao et al. (2009) faz um trabalho que contribui para o tema, buscando na literatura diversos conceitos de risco e concluem que, na área financeira, somente seu aspecto negativo é considerado e percebem que sua definição pode ser entendida como "variação no retorno esperado".

\section{Gerenciamento de Risco: conceito}

Para Cheng et al. (2012) e Norrman et al. (2004), o GR é o processo onde decisões são tomadas no sentido de aceitar, evitar, transferir ou compartilhar um risco reconhecido ou implementar ações para reduzir as consequências ou probabilidade de ocorrência do evento adverso. Dessa mesma forma, Ritchie et al. (2007a), consideram que o objetivo do Gerenciamento de Risco é agir nas três dimensões do risco, já descritas na seção anterior: probabilidade de ocorrência, consequências desse evento adverso ou caminhos que levam a ele.

Blome et al. (2011) amplia o escopo ao afirmar que o GR se trata de um processo a ser aplicado dentro e fora da companhia com o objetivo de identificar potenciais eventos que podem afetá-la e gerenciar o risco de modo a assegurar o alcance dos objetivos da companhia. Já Norrman et al. (2004), detalham os estágios do processo de GR, que consistem em entender o risco e minimizar seu impacto por estágios específicos que variam na literatura entre identificação, análise, avaliação, mitigação e controle, mas que não são unânimes entre os autores.

Khan et al. (2007), partindo desse escopo maior e estágios específicos, conclui, de sua revisão bibliográfica, que o processo de GR deveria ser de contínuo desenvolvimento e que surge da estratégia da empresa, passando por sua implementação e deve abordar metodicamente todos os riscos que cercam as atividades da organização nos tempos presente, passado e, principalmente, futuro. Deve traduzir a estratégia em objetivos táticos e operacionais, atribuindo a responsabilidade por toda a organização.

\section{Modelo de Gerenciamento de Risco}

É consenso entre diversos autores (TRKMAN et al., 2009; HALLIKAS, 2004; TUNCEL et al., 2010; KERN, 2012), que o processo básico de Gerenciamento de Risco consiste em: identificação do risco; análise ou avaliação; e mitigação e controle. Identificação: essa etapa torna os tomadores de decisão conscientes sobre eventos e fenômenos que causam incerteza e seu objetivo principal é reconhecer os riscos futuros, para que a organização esteja apta a gerenciá-lo proativamente (HALLIKAS, 2004). Norrman et al. (2004) concorda sobre a identificação dos riscos e acrescentam que é importante não somente reconhece-los, mas também 
suas causas. Shi, (2004) traz que essa etapa de identificação inicia com uma análise do cenário, com brainstorming que englobe diversos atores na organização e com busca do histórico.

Avaliação: os riscos anteriormente identificados são priorizados, geralmente comparando-se o impacto de suas consequências e probabilidade de ocorrência para definir quais ações serão tomadas, onde se pode usar um mapa de risco como ferramenta auxiliar. Se dados históricos estiverem disponíveis podem e devem ser usados, caso contrário, modelos matemáticos podem auxiliar no trabalho de avaliar probabilidade e impacto (NORRMAN et al., 2004; HALLIKAS, 2004; SHI, 2004).

Mitigação: Hallikas (2004) considera que essa etapa depende diretamente das etapas anteriores que darão a indicação de quais ações tomar e em quais riscos. Os mesmos autores colocam que as estratégias de mitigação podem ser de transferir o risco, de tomá-lo como responsabilidade individual, eliminá-lo, reduzilo ou analisar novamente. Em ambientes de interrelacionamento, como nas CS, o objetivo dessa etapa é que ela tenha caráter colaborativo, entendendo que mitigar esse risco seja benéfico para os demais membros.

Controle: Hallikas (2004) afirma que nem a organização, nem seu ambiente são estáticos, mudam constantemente assim como os riscos e que, por esse motivo, eles devem ser constantemente monitorados. O objetivo é que tendências de crescimento sejam observadas na probabilidade de ocorrência ou no impacto para a organização.

\section{Cadeia de Suprimentos}

\section{Conceito}

Segundo Lambert et al. (2000), atualmente não há competição entre entidades autônomas e sim entre cadeias e o sucesso final de cada empresa depende da sua capacidade de administrar essa complexa rede de relações comerciais. Diversos autores, tais como Ritchie et al. (2007b), Rangel et al. (2014), Neiger et al. (2009), Singhal et al. (2011) e Peck (2005) utilizam uma definição para CS que consiste, basicamente, em uma rede de organizações envolvidas desde o início do fornecimento até a distribuição nos diferentes processos e atividades que agregam valor em forma de produtos ou serviços até o consumidor final. E nessa rede também estão envolvidos os fluxos de informação, fluxos financeiros e desenvolvimento de relacionamentos, fluxos menos tangíveis que os citados primeiramente.

Trkman et al. (2009) corrobora com o fenômeno de competição entre cadeias, dizendo que é preciso compreendê-la não somente como uma relação fornecedor $x$ cliente e sim como uma rede interligada, onde a qualidade, o custo e o risco de um produto ofertado é função dessa rede como um todo. Os mesmos autores ainda afirmam que a tomada de decisão no que concerne à cadeia é diferenciada porque envolve diversos membros, cada um desempenhando um papel e agregando valor ao resultado final e as decisões tomadas repercutem na cadeia como um todo.

\section{Risco na Cadeia de Suprimentos}

Goh et al. (2007) e Tummala et al. (2011) utilizam um conceito próprio para o risco nas CS, afirmando que é a potencial ocorrência de um incidente associado a falhas dos fornecedores que podem resultar da 
incapacidade de atender sua demanda de maneira correta e/ou segura. Pfohl et al. (2011) e Kanyoma (2013) acrescentam que esse atendimento à demanda pode ser em termos de valor entregue, custo, tempo ou qualidade.

De acordo com Shi (2004), as empresas enfrentam riscos quando compram bens de seus fornecedores, quando produzem bens e quando os vendem aos seus consumidores finais. Riscos como os preços dos bens adquiridos e da estratégia de preço ofertada pela empresa, risco de qualidade, riscos de quantidade de estoque e risco relacionado à complexidade da cadeia são algumas das categorias definidas por esse autor a respeito dos riscos enfrentados em uma cadeia de suprimentos, além dos riscos relacionados ao design da cadeia, processos, logística e apoio ao cliente.

Ritchie et al. (2007b) apresentam mais características a esse respeito, afirmando que a quantidade de membros na Cadeia e o nível de interdependência entre esses membros o que reafirma o dito por Shi, (2004) e inclui o termo interdependência, que será bastante falado à frente quando será tratado SCRM. Ainda, conforme Shi (2004), os riscos na CS são de mais difícil identificação, por suas complexas interações e que os riscos em uma Cadeia podem surgir em qualquer lugar, pois eles podem afetar e serem afetados por qualquer processo em qualquer dos membros.

Lavastre et al. (2012) também consideram que é necessário identificar, avaliar e gerenciar esse risco. Norrman et al. (2004), a partir de suas buscas na literatura, encontraram que o risco nas Cadeias pode ser classificado entre riscos de fornecimento e riscos de demanda e acrescentam que essa percepção de risco deve ser ampla, uma vez que uma Cadeia de Suprimentos inclui, no mínimo, três entidades. Alguns autores se preocuparam em classificar os riscos na Cadeia de Suprimentos em seus trabalhos. As classificações são diversas, conforme se aborda no quadro 1.

Quadro 1: Classificação de riscos na cadeia.

\begin{tabular}{|c|l|}
\hline $\begin{array}{c}\text { Autores } \\
\text { (PFOHL et } \\
\text { al., 2011). }\end{array}$ & Riscos dentro da companhia, riscos fora da companhia, mas dentro da Cadeia e riscos fora da Cadeia. \\
\hline $\begin{array}{c}\text { (SINGHAL et } \\
\text { al., 2011). }\end{array}$ & Riscos de características operacionais, de mercado, estratégicas, de produto ou mistas. \\
\hline $\begin{array}{c}\text { (KHAN et al., } \\
\text { 2007) }\end{array}$ & Riscos tecnológicos ou estratégicos. \\
\hline $\begin{array}{c}\text { (WAGNER et } \\
\text { al., 2008) }\end{array}$ & Riscos de demanda; de fornecimento; regulatórios, legais ou burocráticos; de infraestrutura e catastróficos. \\
\hline $\begin{array}{c}\text { (GHADGE et } \\
\text { al., 2012) }\end{array}$ & $\begin{array}{l}\text { Riscos organizacionais, riscos de estoques, riscos de processos ou operacionais, riscos de qualidade, riscos de } \\
\text { relacionamento na rede, riscos do ambiente. }\end{array}$ \\
\hline $\begin{array}{c}\text { (RANGEL et } \\
\text { al., 2014) }\end{array}$ & $\begin{array}{l}\text { Desenvolvimento de 20 classificações de riscos, e identificar 56 tipos de riscos dentro das classificações, para } \\
\text { auxiliar no processo de identificação e possíveis formas mais adequadas de mitigação. As classificações são: fluxo } \\
\text { de controle do ambiente externo, regulatórios e legais, mercado financeiro, capacidade financeira, previsão de } \\
\text { demanda, problemas logísticos, transporte dentro da cadeira, problemas nos sistemas de informação, culturais, } \\
\text { estratégicos, capacidade de produção, infraestrutura, nível de serviço, organizacionais e outros problemas. }\end{array}$ \\
\hline
\end{tabular}

\section{Gestão da Cadeia de Suprimentos}

Para Lambert et al. (2000), o gerenciamento das múltiplas relações no decorrer da CS tem sido referido como a Gestão da Cadeia de Suprimentos, que oferece a oportunidade de capturar a sinergia intra e interorganizacional e lida com a excelência total dos processos do negócio e relações com os outros membros da CS. 
Rao et al. (2009) e Blos (2009) utilizam a definição de que o Gerenciamento da Cadeia é a coordenação estratégia e sistemática das funções e negócios tradicionais dentro e fora de uma companhia, com o objetivo de melhorar o desempenho da Cadeia como um todo. Boyson, (2014) acrescenta que esse processo inclui o gerenciamento das operações de produção e as outras atividades tais como marketing, vendas, design do produto e finanças.

Trkman et al. (2009) trazem uma definição mais amplificada, afirmando que a Gerenciamento da Cadeia de Suprimentos (GCS) é um conjunto de atividades multifuncionais e multidisciplinares que lidam não somente com os atributos e atividades mais físicas e tangíveis, mas os comportamentais e intangíveis também. Ainda, consideram o Gerenciamento um relacionamento e integração proativos entre os vários membros da Cadeia.

De acordo com Shu (2014) e Singhal et al. (2011), o interesse pela GCS iniciou-se na década de 1980. Ao que Tang (2006) reafirma e acrescenta que a provável razão é que, a partir dessa década passa-se a testemunhar as companhias terceirizando suas funções secundárias, o que inclui design, logística, TI de maneira a focar na atividade principal. As companhias perceberam que, esse foco nas funções essenciais e a cooperação permitiam as empresas reduzir custos, reduzir tempo de desenvolvimento de produto e aumentar a qualidade ao mesmo tempo.

Nesse sentido, Boyson, (2014), traz um pequeno histórico do assunto afirmando que em 1982 o termo é cunhado por Booz Allen Hamilton, em 1995 a Universidade de Maryland realiza uma pesquisa com 1300 companhias com o intuito de entender esse interesse da GCS, não somente as iniciativas de integração internas como também as estratégias de integração com clientes e fornecedores. Em 1996 o Supply Chain Council é formado por 69 companhias e desenvolve o um conjunto de práticas e processos nomeado de Supply Chain Operations Reference (SCOR). Em 2002 esse conselho ganha novo nome: Council of Supply Chain Management Professionals (LAMBERT et al., 2000).

Wu (2013) afirma que a CS deve ser gerenciada por inúmeras razões, incluindo sua importância em termos de atendimento à demanda, sua complexidade e os inúmeros eventos a que elas estão suscetíveis e que podem interromper um ou mais de seus fluxos. E, segundo Tang (2006), o Gerenciamento da Cadeia lida com cinco questões principais: design da Cadeia, relação com o fornecedor, processo de seleção do fornecedor, alocação da ordem de fornecimento e contrato de fornecimento.

Trkman et al. (2009) traz que o Gerenciamento da Cadeia não é tarefa fácil em ambientes turbulentos e com rápida mudança. Assegurar que a Cadeia não sofra rompimentos a partir do Gerenciamento da Cadeia se tornou impraticável, quando se enfrenta essa multiplicidade de riscos, como visto na seção anterior. Partindo da preocupação com a interrupção dos fluxos e de que os riscos aos quais a Cadeia está suscetível não são mencionados entre as questões principais do Gerenciamento da Cadeia. Essa é a base para a próxima discussão: o Gerenciamento de Risco da Cadeia de Suprimentos.

Segundo Trkman et al. (2009), uma vantagem competitiva está baseada na habilidade da cadeia de encarar e se adaptar a ambientes de mudança visto que as CS estão expostas a uma grande variedade de 
riscos, como visto anteriormente. E como Shu (2014) salienta, resistência insuficiente da cadeia ou falta de foco em gerenciar os riscos em ambientes de alta complexidade e mudança.

Com as empresas sendo levadas à criação de parcerias que fossem mutuamente benéficas e que se pautassem na cooperação e não somente na competição, as CS foram se estabelecendo e, atualmente a competição, tomou uma dimensão mais sistêmica substituindo a percepção de competição entre companhias isoladas. A partir daí foi sendo necessário que essa cadeia fosse gerenciada, pois tinha características próprias e complexidade muito maiores do que quando se fala de uma única companhia.

Como Pfohl et al. (2011) afirmara, o Gerenciamento de Risco tem um escopo diferente e específico do que o SCRM necessitava, pois a unidade de análise representa uma relação de negócio entre várias empresas sobretudo porque as Cadeias de Suprimentos estão suscetíveis a uma série de riscos que tornava impraticável o Gerenciamento da Cadeia.

Norrman et al. (2004) considera que um fator chave na continuidade e bom desempenho da cadeia é o compartilhamento de riscos e recompensas e, então, surge a necessidade de explorar esse aspecto com mais rigor. Thun et al. (2011) acrescenta que as consequências de rupturas nas cadeias vão além das perdas financeiras e incluem imagem negativa, má reputação, acompanhada de perda de demanda.

Trkman e Mccormack, (2009) afirmam que SCRM é um tema de estudo que vem ganhando importância gradualmente, originado da GCS, quando começam os imperativos teóricos começam a refletir e as necessidades dos profissionais quanto a gerenciar os riscos aos quais a Cadeia está exposta. Ritchie et al. (2007b) ressalta aspectos para o aumento no interesse do assunto: as estratégias e estruturas relativas à cadeia estão evoluindo muito rápido e mudando os formatos em busca de vantagem competitiva. As mudanças tecnológicas apesar de terem seus benefícios, constituem uma ameaça à CS estabelecidas.

Quanto à relevância do tema, Xia et al. (2011) considera que SCRM tem grande influência no estabelecimento de uma cooperação entre os parceiros e no desempenho da cadeia como um todo. Scannell et al. (2013) conclui em seus estudos que uma CS orientada a riscos estabelece uma posição competitiva e benefícios de longo prazo aos seus stakeholders. Adicionalmente, Thun et al. (2011) acrescenta que as consequências de rupturas nas cadeias vão além das perdas financeiras e incluem imagem negativa, má reputação, acompanhada de perda de demanda.

$\mathrm{Na}$ literatura é possível encontrar razões e tendências nos negócios que aumentaram a vulnerabilidade da cadeia. Autores como Zhao (2013), Ghadge et al. (2012) Kern (2012) Lavastre et al. (2012) Ghadge et al. (2012) destaca algumas razões e em seguida é apresentada uma compilação do que é apresentado por eles e outros:

As oportunidades de competir globalmente aumentam a exposição da cadeia e adicionam novas dimensões de riscos: Thun e Hoenig, (2011) afirmam que, as empresas são forçadas pelas tendências atuais a pensarem em mercados globais, tanto em termos de consumidores como de fornecedores. Apesar das oportunidades de receitas e redução nos custos, isso aumenta o nível de complexidade das cadeias e, consequentemente, sua vulnerabilidade a riscos e a dificuldade em gerenciá-la. Pfohl; Köhler e Thomas, (2010) colocam os custos substancialmente maiores de se coordenar cadeias dispersas. Além disso, Jia et al. (2010) acrescentam à discussão os fatores relacionais e a adaptação cultural que configuram mais um risco às cadeias globais. A distância e dispersão entre seus membros, que aumentam os riscos quanto interrupção dos fluxos, por exemplo no transporte 
(GIUNIPERO et al., 2004). Thun et al., (2011) coloca outro fator de grande importância: taxa de câmbio e legislações de importação e exportação;

A interdependência cada vez maior entre seus membros: as parcerias são fatores-chave para as empresas, que podem reduzir custos de transação, se concentrar em suas atividades principais, ter acesso facilitado à tecnologia e informação (HALLIKAS, 2004). Entretanto, Hallikas (2004) salienta riscos da interdependência advinda dessas parcerias, como resistência à mudança, discordância de práticas entre os membros, conflitos de relacionamento. Ademais, há uma necessidade muito maior de sistemas de informação e produção eficientes e confiáveis. Blome et al. (2011) em seu trabalho sobre SCRM em situações de crises financeiras evidenciam como o número de falências entre 2008 e 2009 foi sem precedentes, e uma reação em cadeia, o que eles justificam como sendo consequência dessa interdependência cada vez maior;

O tamanho e complexidade crescentes das cadeias trazem riscos como falta de confiança, assimetria de informações, dependência da terceirização e padronização de contratos;

Terceirizações: Tang (2006) e Thun et al. (2011) afirma que as terceirizações tornam as empresas cada vez mais interdependentes e difíceis de controlar. Como visto anteriormente, acidentes e outros riscos ocorridos com os fornecedores podem causar perdas diretas às empresas. Os mesmos autores citam o exemplo da Land Rover que, em 2001, teve gastos milionários para evitar uma parada na produção por nove meses e perda de 1500 postos de trabalho, causados pela falência de um parceiro;

Estratégias como Lean Six Sigma e Just in Time: Conforme Norrman et al. (2004) e Thun et al. (2011) que essas metodologias se mostraram eficientes e implementá-las passou a ser um fator de status, como única forma de ser competitiva, porém os autores salientam que isso torna as Cadeias mais vulneráveis pois, conforme reafirma Pfohl et al. (2010), não há reservas de materiais ou produtos acabados caso haja qualquer perturbação na Cadeia, o que coloca em risco as receitas da empresa, sua imagem e confiança perante o mercado;

Ciclo de vida dos produtos cada vez menores Zhao (2013) (GHADGE et al., 2012);

Desastres naturais e/ou causados pelo homem: Norrman e Jansson, (2004) afirmam que o foco no gerenciamento do risco da cadeia é tentar evitar efeitos devastadores de desastres ou rompimentos nos fluxos da Cadeia e cita alguns exemplos, tais como o Furacão Floyd na Carolina do Norte (EUA) que inundou plantações e interrompeu o fornecimento de comida por sete dias; um incêndio em 1997 que forçou a Toyota a fechar 18 plantas produtivas por duas semanas, o que custou US\$195mi em perdas de vendas e US\$325mi em veículos e peças. Tang, (2006), traz os exemplos da Ericsson também com incêndio em um fornecedor que teve perdas de US\$400mi e a Apple após um terremoto em Taiwan que interrompeu seu fornecimento de chips. Nejad et al. (2014) cita que os ataques terroristas em 11 de setembro fizeram inúmeras companhias interromperem suas Cadeias, inclusive a Ford que parou a produção em cinco plantas, pela suspensão do tráfego aéreo. Acrescenta-se que, após o 11/09/2001 houve um interesse maior no tema, com aumento de publicações (GHADGE et al., 2012; GIUNIPERO et al., 2004; MENTZER et al., 2008; SHI, 2004), e apresentam sobre as dificuldades enfrentadas pela Nike na implementação do software de Gerenciamento da Cadeia de Suprimentos que levou a uma redução de US\$100mi em receitas, em 2001.

Wieland et al. (2012) considera que a SCRM deve ser entendida tanto para gerenciar os riscos cotidianos aos quais a cadeia está exposta, quanto os extraordinários, como desastres naturais e acidentes. Ainda, o gerenciamento deve ser feito de maneira reativa, monitorando mudanças na cadeia, necessidades dos clientes, tecnologia, estratégias dos parceiros e competidores e os demais riscos que são as fundações para reagir rapidamente aos eventos; e de maneira proativa, identificando os riscos e implementando ações que os previnam ou minimizem os impactos. O gerenciamento de riscos na cadeia ocorre por meio da coordenação ou colaboração entre os parceiros da CS de maneira a aumentar sua rentabilidade e continuidade (TRKMAN et al., 2009; RAO et al., 2009; NORRMAN et al., 2004; TANG, 2006; JIA et al., 2010; BLOS, 2009). 
Lavastre et al. (2012) traz um novo conceito, afirmando que SCRM implica em avaliação, em horizontes estratégicos e operacionais, de curto e longo prazo, de riscos que podem afetar os fluxos de informação, materiais e financeiros e centra-se na agilidade para identificar os riscos previamente ou reagir a eles, diminuindo seus efeitos adversos.

Já Trkman et al. (2009) traz o objetivo da matéria objetivo abordar a identificação, avaliação, análise e tratamento das áreas de vulnerabilidade e risco em CS. Assim como Rao et al. (2009) e Manuj et al. (2014), que fazem uma busca na literatura por conceitos de SCRM e encontram que outros de seus objetivos são reduzir a vulnerabilidade ou aplicar ferramentas de gerenciamento de risco, em colaboração com os demais membros, para lidar com os riscos e incertezas.

Ainda, o objetivo pode ser o de identificar potenciais fontes de risco da Cadeia e implementar ações apropriadas para evitar ou conter a vulnerabilidade da cadeia. Mentzer et al. (2008) ao estudar SCRM em cadeias globais acrescentam que o objetivo, ainda, pode ser reduzir as perdas, a probabilidade ou velocidade do evento adverso, o tempo de detecção desses eventos frequência ou exposição a eles. Kleindorfer et al. (2005) dá uma contribuição ao tema, trazendo os dezessete princípios da matéria, que podem ser entendidos como uma evolução em termos de reconhecimento de sua importância:

O foco do gerenciamento de risco é nas áreas limítrofes da companhia na Cadeia de Suprimentos;

A companhia tem profundo conhecimento das interfaces em sua Cadeia;

Gestão da Cadeia de Suprimentos e SCRM são vistos como integrados e não independentes um do outro;

SCRM interno é coordenado e integrado;

SCRM é parte da estratégia corporativa;

Os gerentes e executivos apoiam e são responsáveis por SCRM;

Todos os membros da Cadeia têm compreensão mútua dos riscos potenciais;

Informações sobre os riscos na Cadeia são disponíveis para toda a companhia;

As companhias dentro de uma Cadeia têm relacionamento cooperativo, justo e próximo;

Companhias dentro de uma Cadeia tem forte confiança mútua;

Assimetrias entre as companhias não existem;

As companhias têm planejamento de processos e metas mútuas para a Cadeia;

As informações sobre riscos são compartilhadas dentro da Cadeia;

Parceiros diretos dentro da Cadeia cooperam entre si quanto à gerenciamento de risco;

Os riscos e recompensas são compartilhados dentro da Cadeia;

Todos os membros (de fornecedores a clientes e de funcionários a executivos) estão envolvidos nas atividades de gerenciamento de risco da Cadeia;

Todos os membros da Cadeia almejam as mesmas metas quanto à Cadeia de Suprimentos.

\section{METODOLOGIA}

Foi realizada uma pesquisa básica qualitativa e bibliográfica quanto aos seus procedimentos. Para tanto foi feita uma busca na base de dados Web of Science, filtrando-se no campo tópico pelo termo 'supply 
chain risk management'. Ao filtrar por esse campo, a busca é realizada por documentos que contenham o termo em seu título, resumo ou palavra-chave.

Ainda, filtrou-se por tipo de documento "artigo" e idioma inglês. O resultado foi a soma de 130 artigos que foram tabulados em uma planilha e organizados por assunto, subdividindo-se em: Risco; Gerenciamento de Risco; Cadeia de Suprimentos; Gerenciamento da Cadeia de Suprimentos; e Gerenciamento de Risco da Cadeia de Suprimentos.

Esses foram os grupos de assuntos identificados na maioria dos artigos e foi base para a construção da revisão bibliográfica de em formato de pirâmide invertida, iniciando-se pelos conceitos de risco até chegar ao Gerenciamento de Risco da Cadeia de Suprimentos. O objetivo foi demonstrar as especificidades de cada um desses assuntos e explicitar o Gerenciamento de Risco da Cadeia como um assunto cujo escopo é diferente dos anteriores. A figura 2 representa a construção da revisão bibliográfica. Foram excluídos os artigos que não tratavam de administração ou economia e para a construção da revisão de maneira coesa e funcional para pesquisadores e profissionais, chegou-se aos artigos aqui referenciados.

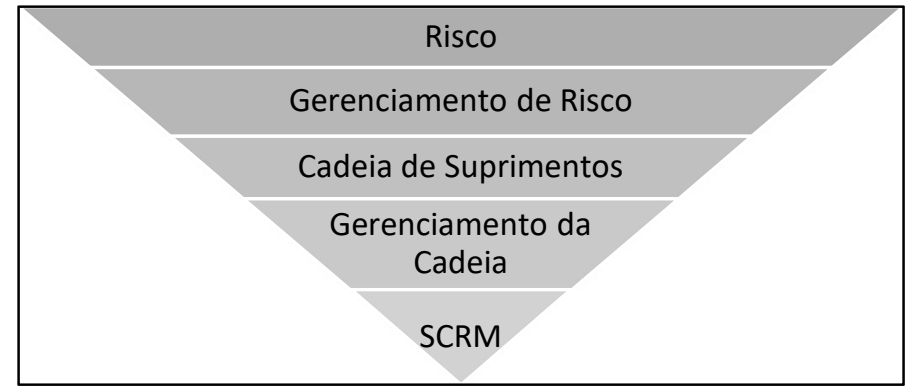

Figura 2: Construção da revisão bibliográfica.

\section{Metodologia para construção de modelos}

Durante a tabulação dos dados, foram sinalizados todos os artigos que traziam em seu conteúdo algum modelo para o processo de Supply Chain Risk Management que foram organizados e serão apresentados. O objetivo era descobrir se o processo é comum e estabelecido para o assunto ou se havia aí uma lacuna a ser apresentada. Por fim, a tabulação foi organizada por data de publicação do artigo, para que ficasse evidente a evolução no assunto e os resultados serão apresentados.

\section{RESULTADOS}

\section{Modelos de SCRM}

Como resultados da revisão bibliográfica, foram realizados dois trabalhos: apresentação dos modelos de gerenciamento de riscos apresentados por diversos autores e um quadro com a evolução histórica do tema, a partir das publicações. Os modelos estão organizados por número de fases e são identificados os autores que apresentaram ou utilizaram cada modelo.

A figura 3 apresenta os autores que utilizam modelos compostos por três fases. Cabe destacar que Shi, (2004) apresenta um modelo de três fases, nos quais ele lista cinco formas de mitigação do risco para a 
última fase: realizar mudanças estratégicas, realizar mudanças organizacionais, operacionais, contratar seguro ou realizar processos de hedge.

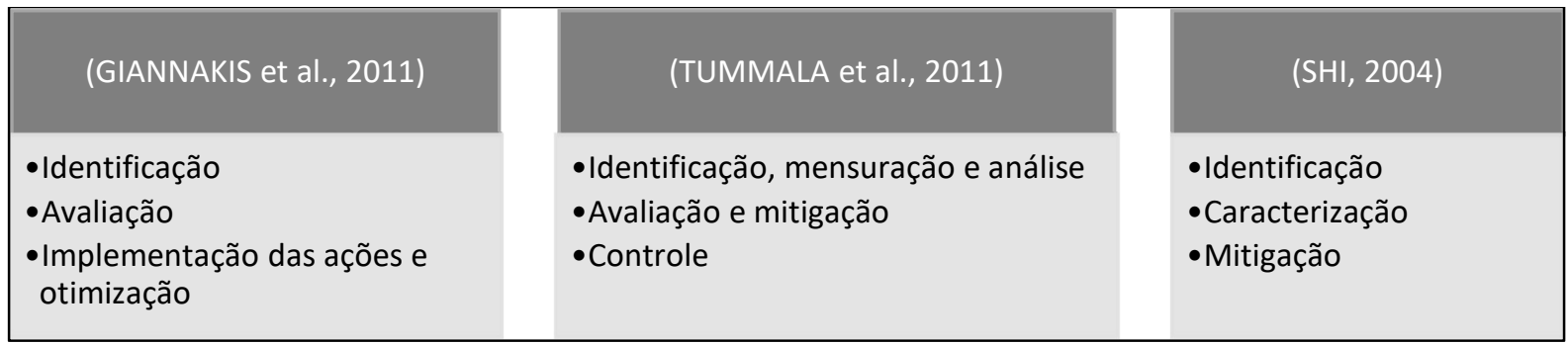

Figura 3: modelos de SCRM com três fases.

O modelo mais comum, utilizado, por exemplo, por Pfohl et al. (2010), Li (2012), Rangel et al. (2014), Blome et al. (2011), Guo (2011), Norrman et al. (2004), Kern (2012) é o modelo de quatro fases apresentado na figura 4 e que está de acordo com o modelo de Gerenciamento de Risco presente na literatura. Já Jia et al. (2010) também utilizam um modelo de quatro fases, mas que difere do descrito anteriormente. Bem como Wu et al. (2006). Lavastre et al. (2012) e Scannell et al. (2013) apresentam modelos com 6 fases, ilustrado pela figura 5. Já Ritchie et al. (2007b) apresentam o modelo com o maior número de fases, como a mostra a figura 6. Já no quadro 2, busca-se apresentar uma linha do tempo dos trabalhos encontrados sobre o tema.

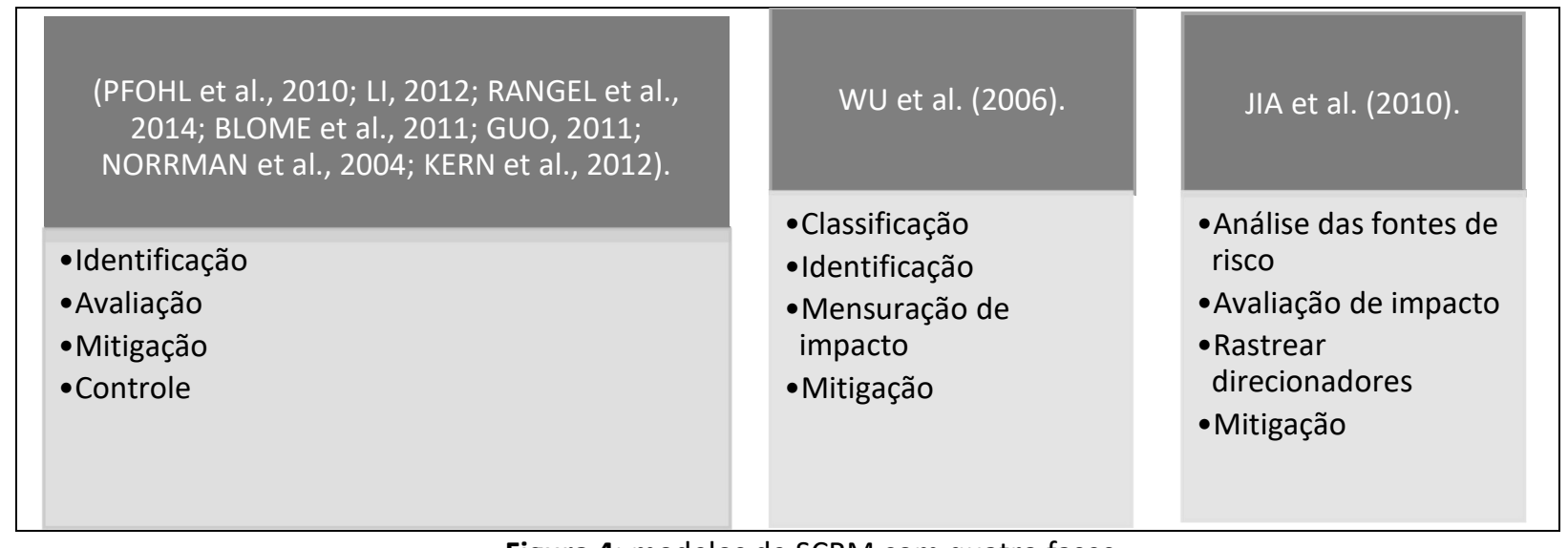

Figura 4: modelos de SCRM com quatro fases.

\begin{tabular}{|c|c|}
\hline (LAVASTRE et al., 2012). & (SCANNELL et al., 2013). \\
\hline $\begin{array}{l}\text { - Mapeamento da Cadeia } \\
\text {-Identificação } \\
\text {-Avaliação } \\
\text { - Desenvolver cenários e situações } \\
\text { dos riscos } \\
\text { - Elaborar estratégia colaborativa } \\
\text {-Implementação }\end{array}$ & $\begin{array}{l}\text { - Estabelecimento de Contexto } \\
\text { - Identificação } \\
\text { - Avaliação } \\
\text { - Tratamento } \\
\text { - Controle }\end{array}$ \\
\hline
\end{tabular}

Figura 5: modelos de SCRM com seis fases. 


\section{(RITCHIE et al., 2007b).}

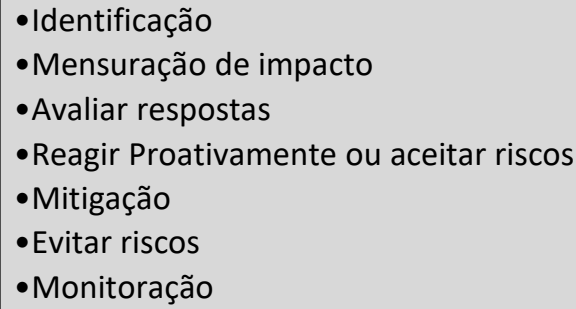

Figura 6: modelos de SCRM com sete fases.

Quadro 2: Evolução histórica do Gerenciamento de Risco da Cadeia de Suprimentos.

\begin{tabular}{|c|c|}
\hline Autores & Assunto \\
\hline (ZSIDISIN 2004). & $\begin{array}{l}\text { Os autores examinam ferramentas e técnicas para empresas avaliarem risco em suas atividades de } \\
\text { compra, buscando a literatura de risco da Cadeia e avaliação de risco. }\end{array}$ \\
\hline (HALLIKAS, 2004). & $\begin{array}{l}\text { Autores mostram desafios que redes de cooperação trazem ao gerenciamento de risco e os resultados } \\
\text { mostram que o gerenciamento de risco é um importante alvo em desenvolvimento nos estudos de Cadeia } \\
\text { de Suprimentos, afirmando que quando a dependência entre companhias aumenta, elas se tornam mais } \\
\text { expostas ao risco. }\end{array}$ \\
\hline $\begin{array}{l}\text { (NORRMAN et al., } \\
\text { 2004). }\end{array}$ & $\begin{array}{l}\text { O objetivo do trabalho é descrever como a Ericsson implementou processos e ferramentas para Gerenciar } \\
\text { o Risco na Cadeia de Suprimentos após um incêndio em um fornecedor interromper o fluxo de materiais e } \\
\text { causar grandes perdas. }\end{array}$ \\
\hline $\begin{array}{l}\text { (KLEINDORFER et } \\
\text { al., 2005). }\end{array}$ & $\begin{array}{l}\text { Autores realizam estudo sobre SCRM na indústria química dos Estados Unidos, considerando acidentes } \\
\text { ocorridos entre } 1995 \text { e } 2000 \text { que provocaram ruptura na Cadeia. }\end{array}$ \\
\hline (PECK, 2005). & $\begin{array}{l}\text { Autor propões quatro níveis de análise para SCRM: cadeia de valor do produto/processo, dependência de } \\
\text { ativos e infraestrutura, redes intra e interorganizacionais e ambiente natural e social. }\end{array}$ \\
\hline (TANG, 2006). & $\begin{array}{l}\text { Desenvolvem uma estrutura para classificação de artigos sobre SCRM, objetivando ter um guia para } \\
\text { futuras pesquisas do tema }\end{array}$ \\
\hline $\begin{array}{c}\text { (WAGNER et al., } \\
\text { 2006). }\end{array}$ & $\begin{array}{l}\text { Estudo com } 760 \text { executivos de companhias alemãs sobre dependência entre alguns fornecedores e } \\
\text { clientes, estratégia de único fornecedor, confiança entre os membros da Cadeia e relação entre esses } \\
\text { temas e vulnerabilidade da Cadeia. }\end{array}$ \\
\hline $\begin{array}{l}\text { (RITCHIE et al., } \\
\text { 2007b). }\end{array}$ & $\begin{array}{l}\text { Desenvolvem uma estrutura de mensuração composta de cinco componentes: direcionadores de risco, } \\
\text { influenciadores de gerenciamento de risco, características dos tomadores de decisão e consequências do } \\
\text { gerenciamento para o desempenho. }\end{array}$ \\
\hline $\begin{array}{l}\text { (GOH et al., } \\
\text { 2007). }\end{array}$ & $\begin{array}{l}\text { Apresenta um modelo estocástico do problema dos multiestágios das cadeias de suprimentos globais, com } \\
\text { desenvolvimento de um algoritmo para maximização de lucros. }\end{array}$ \\
\hline $\begin{array}{l}\text { (RITCHIE et al., } \\
\text { 2007a). }\end{array}$ & $\begin{array}{l}\text { Examinam os modelos existentes na literatura e exploram sua aplicação no contexto da Cadeia de } \\
\text { Suprimentos. }\end{array}$ \\
\hline $\begin{array}{l}\text { (KHAN et al., } \\
\text { 2007). }\end{array}$ & Revisão de literatura sobre o tema e relacionando à literatura tradicional de risco. \\
\hline $\begin{array}{l}\text { (WAGNER et al., } \\
\text { 2008). }\end{array}$ & $\begin{array}{l}\text { O trabalho busca prover detalhes de fontes de risco na Cadeia de Suprimentos, discutindo com } \\
\text { especialistas e profissionais com o objetivo de relacionar essas fontes de risco ao desempenho da Cadeia } \\
\text { de Suprimentos. }\end{array}$ \\
\hline $\begin{array}{l}\text { (MENTZER et al., } \\
\text { 2008). }\end{array}$ & Artigo apresenta um modelo de mitigação de risos para Cadeia de Suprimentos Globais. \\
\hline $\begin{array}{l}\text { (TRKMAN et al., } \\
\text { 2009). }\end{array}$ & Propõem modelo de avaliação de risco do fornecedor, baseado em quatro pilares. \\
\hline $\begin{array}{l}\text { (HENDRICKS et al., } \\
\text { 2009). }\end{array}$ & $\begin{array}{l}\text { Examinam se diversificação de negócios, diversidade geográfica e verticalização impactam em rupturas da } \\
\text { Cadeia. Estudo com } 307 \text { anúncios de rupturas da Cadeia. }\end{array}$ \\
\hline $\begin{array}{l}\text { (NYOMAN et al., } \\
\text { 2009). }\end{array}$ & $\begin{array}{l}\text { Autores desenvolvem um modelo de gerenciamento do risco em SC, que permite que as companhias } \\
\text { selecionem os riscos a serem tratados de acordo com a relevância. }\end{array}$ \\
\hline (BLOS, 2009). & $\begin{array}{l}\text { Estudo exploratório em indústrias automotivas e eletrônicas no Brasil, com o objetivo de identificar os } \\
\text { riscos de suas cadeias e verificar a necessidade de implementação de um gerenciamento desses riscos. }\end{array}$ \\
\hline (JIA et al., 2010). & $\begin{array}{l}\text { Buscam acrescentar uma nova discussão à literatura de SCRM: relação cultural. Fazendo uma pesquisa } \\
\text { entre as diferenças e as formas de adaptação cultural de companhias chinesas com companhias ocidentais } \\
\text { e a relação com riscos na Cadeia. }\end{array}$ \\
\hline $\begin{array}{l}\text { (PFOHL et al., } \\
\text { 2010). }\end{array}$ & $\begin{array}{l}\text { Autores fazem uma revisão da literatura e, como contribuição para o campo, propõem } 17 \text { princípios de } \\
\text { Gerenciamento de Risco da Cadeia de Suprimentos. }\end{array}$ \\
\hline (GUO, 2011). & Desenvolvem um modelo sistemático baseado na literatura sobre o tema como uma para gerentes. \\
\hline $\begin{array}{l}\text { (BLOME et al., } \\
\text { 2011). }\end{array}$ & $\begin{array}{l}\text { Autores investigam experiências bem-sucedidas de companhias que buscaram gerenciar o risco. Estudo de } \\
\text { caso com oito companhias europeias. }\end{array}$ \\
\hline $\begin{array}{l}\text { (SINGHAL et al., } \\
\text { 2011). }\end{array}$ & $\begin{array}{l}\text { Autores realizam um estudo empírico de fontes e direcionadores de riscos na Cadeia na indústria } \\
\text { Aeroespacial. }\end{array}$ \\
\hline
\end{tabular}




\begin{tabular}{|c|c|}
\hline $\begin{array}{l}\text { (THUN et al., } \\
\text { 2011). }\end{array}$ & $\begin{array}{l}\text { Autores investigam empiricamente a prática de SCRM, a partir de survey com } 67 \text { companhias automotivas } \\
\text { alemãs, identificando seus riscos e, em seguida avaliando sua probabilidade e impacto. }\end{array}$ \\
\hline $\begin{array}{l}\text { (CHENG et al., } \\
\text { 2012). }\end{array}$ & $\begin{array}{l}\text { Os autores exploram SCRM por meio da abordagem relacional no mercado chinês e desenvolvem um } \\
\text { modelo teórico baseado no capital social. }\end{array}$ \\
\hline (KERN, 2012). & $\begin{array}{l}\text { Examinam como ferramentas e métodos prévios de gerenciamento de risco podem contribuir com o } \\
\text { desempenho. }\end{array}$ \\
\hline $\begin{array}{l}\text { (HAHN et al., } \\
\text { 2012). }\end{array}$ & $\begin{array}{l}\text { Autores desenvolvem uma estrutura para avaliação de risco e desempenho financeiro e aplicação em uma } \\
\text { companhia com comportamento averso ao risco. }\end{array}$ \\
\hline $\begin{array}{l}\text { (GHADGE et al., } \\
\text { 2012). }\end{array}$ & $\begin{array}{l}\text { Autores examinam a literatura e os modelos de gerenciamento de risco propostos, no intuito de identificar } \\
\text { mudanças estratégicas no campo. }\end{array}$ \\
\hline $\begin{array}{l}\text { (LAVASTRE et al., } \\
\text { 2012). }\end{array}$ & $\begin{array}{l}\text { Estudo empírico com } 142 \text { gerentes de } 50 \text { companhias francesas para buscar a importância do SCRM ser } \\
\text { uma função gerencial e interorganizacional, muito próxima das realidades estratégicas e operacionais. }\end{array}$ \\
\hline (WU, 2013). & $\begin{array}{l}\text { Autores buscam entender o impacto de ruptura de fornecimento impactam a cadeia, considerando market } \\
\text { share do produtor e do distribuidor. }\end{array}$ \\
\hline $\begin{array}{l}\text { (KANYOMA, } \\
\text { 2013). }\end{array}$ & $\begin{array}{l}\text { Estudo de caso em um hospital público do Malaui, abordando a falha de fornecimento e a estratégia de } \\
\text { fornecedor único, além das consequências desta para a Cadeia de Suprimentos. }\end{array}$ \\
\hline $\begin{array}{l}\text { (TAKATA et al., } \\
\text { 2013). }\end{array}$ & $\begin{array}{l}\text { Autores propõem um método para avaliar os potenciais riscos associados aos Bom sem fornecedores } \\
\text { identificados. }\end{array}$ \\
\hline $\begin{array}{l}\text { (CHEN et al., } \\
\text { 2013). }\end{array}$ & $\begin{array}{l}\text { Autores propõem uma modificação ao método FMEA para seleção de fornecedores pela ótica do } \\
\text { Gerenciamento de Risco da Cadeia de Suprimentos. }\end{array}$ \\
\hline $\begin{array}{l}\text { (TAZELAAR et al., } \\
\text { 2013). }\end{array}$ & $\begin{array}{l}\text { Estudo centra-se em uma revisão de literatura e experiência com dois grupos focais: membros do Supply } \\
\text { Chain Thougt Leaders e do International SCRM. }\end{array}$ \\
\hline (BOYSON, 2014). & $\begin{array}{l}\text { Autor trata de uma nova disciplina: Cyber Supply Chain Risk Management, criada para ajudar os executivos } \\
\text { de TI nos desafios da rápida globalização e difusão da terceirização de sistemas de hardware e software. }\end{array}$ \\
\hline $\begin{array}{l}\text { (NEJAD et al., } \\
\text { 2014). }\end{array}$ & $\begin{array}{l}\text { Autores desenvolvem uma ferramenta de apoio à decisão para resposta apropriada quando da } \\
\text { necessidade de executar um plano de contigência em grandes rupturas da Cadeia e desastres naturais. }\end{array}$ \\
\hline $\begin{array}{l}\text { (RANGEL et al., } \\
\text { 2014). }\end{array}$ & $\begin{array}{l}\text { Pesquisa que categoriza } 20 \text { classificações de risco da Cadeia, incluindo } 56 \text { tipos de risco ao total, com o } \\
\text { objetivo de contribuir com a literatura e auxiliar no gerenciamento do risco na Cadeia de Suprimentos. }\end{array}$ \\
\hline $\begin{array}{l}\text { (OLIVEIRA et al., } \\
\text { 2014). }\end{array}$ & $\begin{array}{l}\text { Artigo explora os riscos aos quais a indústria automotiva no Brasil está exposta e objetiva compreendê-los } \\
\text { para contribuir com atividades de tratamento e controle dos riscos operacionais. }\end{array}$ \\
\hline $\begin{array}{l}\text { (AMBULKAR et al., } \\
\text { 2015). }\end{array}$ & $\begin{array}{l}\text { O artigo explora os fatores que contribuem para o aumento de resiliência da Cadeia às rupturas causadas } \\
\text { por desastres naturais. }\end{array}$ \\
\hline $\begin{array}{l}\text { (BODE et al., } \\
\text { 2015). }\end{array}$ & Trabalho sobre fatores que aumentam a frequência de ruptura na Cadeia. \\
\hline (CHAE, 2015). & $\begin{array}{l}\text { Estudo analítico na rede social Twitter sobre Gerenciamento da Cadeia e Gerenciamento de Risco da } \\
\text { Cadeia para avaliar o potencial dessa e outras redes sociais nas práticas de SCM e SCRM. }\end{array}$ \\
\hline
\end{tabular}

\section{DISCUSSÃO}

Após a revisão bibliográfica realizada, é possível inferir algumas considerações sobre o SCRM enquanto tema relativamente novo de estudos. Sua importância é evidenciada com as razões e acontecimentos trazidos pelos autores e comprovam que o Gerenciamento da Cadeia de Suprimentos não contempla essa análise, sendo necessário levá-lo e trata-lo nas Cadeias também. Ainda sobre os motivos apresentados na literatura, é possível que, a partir deles se faça uma análise nas empresas sobre quais dos citados influenciam mais a Cadeia e essa seja uma forma de identificar os riscos preliminarmente.

Quanto ao seu conceito, é consenso que a confiança e a conexão entre os membros da Cadeia precisa ser fortalecida e, independente da estratégia e estrutura de Gerenciamento do Risco na Cadeia, é necessária uma abordagem proativa para eficiência. A evolução da disciplina é clara, o trabalho de descrever algumas publicações e sua contribuição ao tema teve o objetivo também de auxiliar os pesquisadores sobre o tema em suas pesquisas e a partir desse tópico foi possível ver como evoluiu em demonstrar a necessidade de se estudar esse assunto, até formas e campos diversos $\mathrm{m}$ que pode ser aplicado, como das no contexto das redes sociais.

No quadro 2 foi possível verificar essa evolução, como por exemplo nas primeiras publicações em 2004 , onde os objetivos foram examinar técnicas e descrever os riscos. A partir de 2006 , vê-se que os autores 
buscaram desenvolver metodologias, realização de estudos de caso em grandes empresas, têm-se uma primeira revisão bibliográfica sobre o tema. Em 2008 e 2009 muitas estruturas e modelos propostos pelos autores para a prática de SCRM. Com a evolução do tema, em 2010 uma nova discussão acrescentada: relação cultural em cadeias globais. Em 2011 muitos estudos empíricos e pesquisas, inclusive um que busca encontrar resultados das práticas de SCRM implementadas em organizações europeias.

A partir de 2013, os autores buscam posicionar o assunto em contextos específicos. Um estudo que contribui para o tema é o que compreendeu um hospital público do Malaui, mostrando os impactos dos riscos da Cadeia e como SCRM pode contribuir nesses casos. O ano de 2014 assinala mais evoluções para o tema, com um trabalho que introduz um novo conceito: Cyber Supply Chain Risk Management e uma pesquisa que apresenta 20 categorias de risco, somando 56 tipos de risco ao total. Por fim, o ano de 2015 se caracteriza por publicações sobre fatores que aumentam a resiliência das CS e um estudo que envolve a rede social Twitter e seu potencial para auxiliar os pesquisadores do tema.

Quanto aos modelos de SCRM, há uma falta de concordância entre os autores quanto à quantidade de fases e quais são elas, com diversos autores apresentando modelos com 3, 4, até 7 fases, sem que cada uma seja necessariamente igual à outra. Esse é um ponto o qual pode ser trabalhado e serve como sugestão para futuras publicações, como forma de evolução da disciplina e seu estabelecimento entre os acadêmicos e profissionais.

O acréscimo de uma quarta fase, a de controle, está de acordo com a literatura tanto de gerenciamento de risco, como de cadeia de suprimentos que salientam a abordagem preventiva dos riscos, em virtude de mitiga-los. Outro ponto de discordância entre os autores é quanto à classificação de riscos. 0 artigo apresentou alguns dos autores que tratam da classificação, que não são os únicos, há outros que apresentam outras classificações, mas não era o objetivo do trabalho esgotar esse ponto da literatura. Entretanto, foi possível ver como é diversa a literatura a esse respeito.

O trabalho de Kanyoma (2013) por tratar do tema no âmbito da Administração pública. Poucos autores o fazem e a partir desse trabalho foi possível ver os níveis a que a contribuição que SCRM pode chegar, inclusive em termos sociais, como no abastecimento de hospitais públicos. Escolas públicas e demais organizações estatais podem se beneficiar com um estudo como esse, inclusive no Brasil. Sobre o Brasil, fica clara a falta de publicações sobre o tema, sendo encontrado um único artigo de Oliveira et al. (2014), que trata especificamente dos riscos operacionais na Cadeia. Espera-se que esse trabalho contribua para o tema no país e que auxilie para o surgimento de outros.

\section{CONCLUSÕES}

O artigo apresentou uma revisão bibliográfica, trazendo os conceitos de Risco e seu histórico dicotômico de ser reconhecido somente como perda ou oportunidade para as empresas e sua importância no contexto gerencial. Em seguida mostrou o modelo de Gerenciamento do Risco com quatro fases para em seguida trazer os conceitos de Cadeia de Suprimentos e Gerenciamento da Cadeia de Suprimentos. 
O referencial bibliográfico sobre Gerenciamento da Cadeia de Suprimentos contemplou o Gerenciamento do Risco na Cadeia para, em seguida apresentar a relevância de se gerenciar esse risco na Cadeia de Suprimentos, como elas estão expostas a esses riscos e o que faz (ou fez) com que ela se tornasse tão vulnerável ao longo do tempo.

Com a revisão bibliográfica realizada, o histórico do Gerenciamento de Risco da Cadeia de Suprimentos, os artigos que contribuem para a evolução do tema e a compilação dos modelos de Gerenciamento apresentados, o trabalho coloca o assunto em pauta e o torna mais explícito aos pesquisadores e profissionais. As sugestões de mais pesquisas do tema no âmbito do setor público e o aumento de pesquisas no Brasil são reiteradas e constituem proposições para a agenda da matéria para o futuro.

\section{REFERÊNCIAS}

AMBULKAR, S.; BLACKHURST, J.; GRAWE, S.. Firm's resilience to supply chain disruptions: Scale development and empirical examination. Journal of Operations Management, v.34, p.111-122, 2015.

BLOME, C.; SCHOENHERR, T.. Supply chain risk management in financial crises-A multiple case-study approach. International Journal of Production Economics, v.134, n.1, p.43-57, 2011.

BLOS, M. F.. Supply chain risk management (SCRM): a case study on the automotive and electronic industries in Brazil. Supply Chain Management: An International Journal, v.14, n.4, p.247-252, 2009.

BODE, C.; WAGNER, S. M.. Structural drivers of upstream supply chain complexity and the frequency of supply chain disruptions. Journal of Operations Management, v.36, p.215-228, 2015.

BOYSON, S.. Cyber supply chain risk management: Revolutionizing the strategic control of critical IT systems. Technovation, v.34, n.7, p.342-353, 2014.

CHAE, B. K.. Insights from hashtag \#supplychain and Twitter Analytics: Considering Twitter and Twitter data for supply chain practice and research. International Journal of Production Economics, v.165, p.247-259, 2015.

CHEN, P. S.; WU, M. T.. A modified failure mode and effects analysis method for supplier selection problems in the supply chain risk environment: a case study. Computers \& Industrial Engineering, v.66, n.4, p.634-642, 2013.

CHENG, T. C. E.; YIP, F. K.; YEUNG, A. C. L.. Supply risk management via guanxi in the Chinese business context: The buyer's perspective. International Journal of Production Economics, v.139, n.1, p.3-13, 2012.

FUNO, K. A.; MUNIZ JUNIOR, J.; MARINS, F. A. S.. Fatores de risco em cadeia de suprimentos do setor aeroespacial: aspectos qualitativos e quantitativos. Production Journal, v.23, n.4, p.832-845, 2013.
GHADGE, A.; DANI, S.; KALAWSKY, R.. Supply chain risk management: present and future scope. International Journal of Logistics Management, v.23, n.3, p.313-339, 2012.

GIANNAKIS, M.; LOUIS, M.. A multi-agent based framework for supply chain risk management. Journal of Purchasing and Supply Management, v.17, n.1, p.23-31, 2011.

GIUNIPERO, L. C.; ELTANTAWY, R. A.. Securing the upstream supply chain: a risk management approach. International Journal of Physical Distribution \& Logistics Management, v.34, n.9, p.698-713, 2004.

GOH, M.; LIM, J. Y. S.; MENG, F.. A stochastic model for risk management in global supply chain networks. European Journal of Operational Research, v.182, n.1, p.164-173, 2007.

GUO, Y.. Research on Knowledge-Oriented Supply Chain Risk Management System Model. Journal of Management and Strategy, v.2, n.2, p.72-77, 2011.

HAHN, G. J.; KUHN, H.. Value-based performance and risk management in supply chains: a robust optimization approach. International Journal of Production Economics, v.139, n.1, p.135-144, 2012.

HALLIKAS, J.. Risk management processes in supplier networks. International Journal of Production Economics, v.90, p.47-58, 2004.

HENDRICKS, K. B.; SINGHAL, V. R.; ZHANG, R.. The effect of operational slack, diversification, and vertical relatedness on the stock market reaction to supply chain disruptions. Journal of Operations Management, v.27, n.3, p.233-246, 2009.

HITT, M. A.; IRELAND, R. DUANE, E.; HOSKISSON, R.. Administração Estratégica. 2 ed. São Paulo: Cengage Learning, 2008.

JIA, F.; RUTHERFORD, C. Mitigation of supply chain relational risk caused by cultural differences between China and the West. The International Journal of Logistics Management, 
v.21, n.2, p.251-270, 2010.

KANYOMA, K. E.. Sourcing Strategy and Supply Chain Risk Management in the Healthcare Sector: A Case Study of Malawi's Public Healthcare Delivery Supply Chain. Journal of Management and Strategy, v.4, n.3, p.16-26, 2013.

KERN, D.. Supply risk management: model development and empirical analysis. International Journal of Physical Distribution \& Logistics Management, v.42, n.1, p.60-82, 2012.

KHAN, O.; BURNES, B.. Risk and supply chain management: creating a research agenda. The International Journal of Logistics Management, v.18, n.2, p.197-216, 2007.

KLEINDORFER, P. R.; SAAD, G. H.. Managing Disruption Risks in Supply Chains. Production and Operations Management, v.14, n.1, p.53-68, 2005.

LAMBERT, D. M.; COOPER, M. C.. Issues in Supply Chain Management. Industrial Marketing Management, v.29, p.65-83, 2000.

LAVASTRE, O.; GUNASEKARAN, A.; SPALANZANI, A.. Supply chain risk management in French companies. Decision Support Systems, v.52, n.4, p.828-838, 2012.

LI, T.. Risk Assessment in the Supply Chain Management Based on Fuzzy AHP Model. Progress in Applied Mathematics, v.4, n.1, p.9-13, 2012.

MANUJ, I.; ESPER, T. L.; STANK, T. P.. Supply Chain Risk Management Approaches Under Different Conditions of Risk: Manuj. Journal of Business Logistics, v.35, n.3, p.241258, 2014.

MENTZER, J. T.; MANUJ, I.. Global Supply Chain Risk Management. Journal of Business Logistics, v.29, n.1, p.133155, 2008.

NEIGER, D.; ROTARU, K.; CHURILOV, L.. Supply chain risk identification with value-focused process engineering. Journal of Operations Management, v.27, n.2, p.154-168, 2009.

NEJAD, A. E.; NIROOMAND, I.; KUZGUNKAYA, O.. Responsive contingency planning in supply risk management by considering congestion effects. Omega, v.48, p.19-35, 2014.

NORRMAN, A.; JANSSON, U.. Ericsson's proactive supply chain risk management approach after a serious sub-supplier accident. International Journal of Physical Distribution \& Logistics Management, v.34, n.5, p.434-456, 2004.

NYOMAN PUJAWAN, I.; GERALDIN, L. H.. House of risk: a model for proactive supply chain risk management. Business Process Management Journal, v.15, n.6, p.953-967, 2009.

OLIVEIRA, U. R.; ROCHA, H. M.. Gerenciamento de riscos operacionais em montadoras de veículos. Pretexto, Belo Horizonte, v.15, n.4, p.27-45, 2014.

PECK, H.. Drivers of supply chain vulnerability: an integrated framework. International Journal of Physical Distribution \& Logistics Management, v.35, n.4, p.210-232, 2005.

PFOHL, H. C.; KÖHLER, H.; THOMAS, D.. State of the art in supply chain risk management research: empirical and conceptual findings and a roadmap for the implementation in practice. Logistics Research, v.2, n.1, p.33-44, 2010.

PFOHL, H.; GALLUS, P.; THOMAS, D.. Interpretive structural modeling of supply chain risks. International Journal of Physical Distribution \& Logistics Management, v.41, n.9, p.839-859, 2011.

RANGEL, D. A.; OLIVEIRA, T. K.; LEITE, M. S. A.. Supply chain risk classification: discussion and proposal. International Journal of Production Research, p.1-20, 2014.

RAO, S.; GOLDSBY, T. J.. Supply chain risks: a review and typology. International Journal of Logistics Management, v.20, p.97-123, 2009.

RITCHIE, B.; BRINDLEY, C.. An emergent framework for supply chain risk management and performance measure. The Journal of the Operational Research Society, v.58, n.11, p.1398-1411, 2007b.

RITCHIE, B.; BRINDLEY, C.. Supply chain risk management and performance. International Journal of Operations \& Production Management, v.27, n.3, p.303-322, 2007a.

SCANNELL, T.; CURKOVIC, S.; WAGNER, B.. Integration of ISO 31000:2009 and Supply Chain Risk Management. American Journal of Industrial and Business Management, v.3, n.4, p.367-377, 2013.

SHI, D.. A review of enterprise supply chain risk management. Journal of Systems Science and Systems Engineering, v.13, n.2, p.219-244, 2004.

SHU, T.. GBOM-oriented management of production disruption risk and optimization of supply chain construction. Expert Systems with Applications, v.41, n.1, p.59-68, 2014.

SILVA, C. A. B.; SOUZA FILHO, H. M. Guidelines for rapid appraisals of agrifood chain performance in developing countries. Rome: FAO, 2007.

SINGHAL, P.; AGARWAL, G.; MITTAL, M. L.. Supply chain risk management: review, classification and future research directions. International Journal of Business Science and Applied Management, v.6, n.3, p.15-42, 2011.

TAKATA, S.; YAMANAKA, M.. BOM based supply chain risk management. CIRP Annals - Manufacturing Technology, v.62, n.1, p.479-482, 2013.

TANG, C. S.. Perspectives in supply chain risk management. International Journal of Production Economics, v.103, p.451-488, 2006.

TAZELAAR, F.; SNIJDERS, C.. Operational risk assessments by supply chain professionals: Process and performance. Journal of Operations Management, v.31, n.1-2, p.37-51, 2013.

THUN, J. H.; HOENIG, D.. An empirical analysis of supply chain risk management in the German automotive industry. International Journal of Production Economics, v.131, n.1, p.242-249, 2011.

TRKMAN, P.; MCCORMACK, K.. Supply chain risk in turbulent 
environments: A conceptual model for managing supply chain network risk. International Journal of Production Economics, v.119, n.2, p.247-258, 2009.

TUMMALA, R.; SCHOENHERR, T.. Assessing and managing risks using the Supply Chain Risk Management Process (SCRMP). Supply Chain Management: An International Journal, v.16, n.6, p.474-483, 2011.

TUNCEL, G.; ALPAN, G.. Risk assessment and management for supply chain networks: A case study. Computers in Industry, v.61, n.3, p.250-259, 2010.

WAGNER, S. M.; BODE, C.. An empirical examination of supply chain performance along by. Journal of Business Logistics, v.29, n.1, p.307-325, 2008.

WAGNER, S. M.; BODE, C.. An empirical investigation into supply chain vulnerability. Journal of Purchasing and Supply Management, v.12, n.6, p.301-312, 2006.

WIELAND, A.; WALLENBURG, C. M.. Dealing with supply chain risks. International Journal of Physical Distribution \& Logistics Management, v.42, n.10, p.887-905, 2012.
WU, T.. Supply chain risk management: An agent-based simulation to study the impact of retail stockouts. IEEE Transactions on Engineering Management, v.60, n.4, p.676686, 2013.

WU, T.; BLACKHURST, J.; CHIDAMBARAM, V.. A model for inbound supply risk analysis. Computers in Industry, v.57, n.4, p.350-365, 2006.

XIA, D.; CHEN, B.. A comprehensive decision-making model for risk management of supply chain. Expert Systems with Applications, v.38, n.5, p.4957-4966, 2011.

ZHAO, L.. The impact of supply chain risk on supply chain integration and company performance: a global investigation. Supply Chain Management: An International Journal, v.18, n.2, p.115-131, 2013.

ZSIDISIN, G. A.. An analysis of supply risk assessment techniques. International Journal of Physical Distribution \& Logistics Management, v.34, n.5, p.397-413, 2004. 